\title{
Special Issue on Soft OR and Complex Societal Problems
}

\author{
Preface by the guest editor
}

It is really troublesome to show how formal planning support tools can contribute to deal with large scale public problems that appear in education, health, safety, human development and infrastructure services.

Rational analysts find great difficulties to construct models to help making decisions about public problems. To the point that either rational or social and political analysts are sceptical about the possibility and efficacy of formal models implementation.

However, some OR researchers have been facing the challenge under the designation of Soft Operational Research, also known as Problem Structuring Methods, to deal with Complex Societal Problems.

This issue of Pesquisa Operacional, the journal of the Brazilian Society of Operations Research, is intended to meet a demand on methods to help thinking and modelling such severe and critical social problems.

Validation is often a missing link in formal multidisciplinary modelling. While formal models should be both exhaustive and parcimonious, when it comes to get sound applied results to support policy making, this is a complex task. Results validation requires seeing beyond the model, i.e. not what the usual models can account for, but what they ignore. This is not attractive to the specialist, since the unknown can hardly be structured. On the other hand, it is worthy identifying the many real aspects of the problem, since "to know what you don't know" leaves you in a better position than "not knowing what you don't know".

Maybe this spirit impregnates the eight papers selected for this issue. Still they range from more specific and delimited problems, the first half, to methodological, perhaps innovative themes that appear in the second half of the issue.

Namen, Bornstein and Rosenhead apply Robustness Analysis for structuring the problems and planning actions in a poor Brazilian Community dedicated to organic farming. Taking sustainability as the organizing principle, the authors report improvements in education, health and sanitary conditions, as well as self-esteem and self-confidence.

Hipel, Hegazy and Yousefi present an innovative multimethodology for strategic and tactic decision making to resolve conflicts in brownfield redevelopment. They resort to Graph Model at the strategic level and utility theory at the tactical level, which could be implemented as a negotiation decision support system.

Melo, Cavalcanti Netto, Ferreira Filho and Fernandes apply an organization analysis methodology for knowledge capitalization to manage the transport of indivisible exceptional cargo in a large electric sector firm. The method succeeded in integrating the stakeholders' perspectives, activity stages and information flows and improving capitalized knowledge on materials, operations and management. 
Gomes, Rangel and Jerônimo provide an illustrative case for the application of cognitive mapping to identify the principal factors that influence the decision of professionals to move from the headquarters of a large corporation in Rio de Janeiro to a business unit outside Rio.

Yolles describes the epistemological positions for OR, the dynamics for viable systems and options for paradigmatic change toward more complex sociocultural problem situations. Although his theoretical framework is nowadays a skeleton, as he states, new data acquisition techniques will enable "Knowledge Cybernetics to interpret the outputs and convert them into intervention strategies".

Lobo and Lins explore non fortuitous parallels between paradigmatic changes in OR and epidemiology/health care. Amongst the recommendations, the authors suggest that health performance assessment through Data Envelopment Analysis should benefit from a systematic interdisciplinary practice in order to enrich health services planning and epidemiological knowledge.

DeTombe advances her research on validation and complex societal problems, focusing on global safety, which concerns risky rare events, but also massive daily mismanagement that surface in the collapse of credit and the crisis in the health systems. According to DeTombe "there are yearly 23000 unnecessary injuries from the wrong treatments in the hospitals... These errors lead to 1700 avoidable deaths" in The Netherlands' health system, not to mention inconsiderate market driven health systems like the US one and those in impoverished countries.

Vidal contributes with a very innovative paper on the relationship amongst creativity, responsible freedom, motivation and self-reflection. His creativity courses develop not in a exclusive OR environment, but in a problem solving setting. Still OR modelling always demands innovative solutions.

We hope in the end that the papers herein will contribute to provide an articulation between the conventional "locally applied" methods in OR to the "globally thinking" social frameworks provided by problem structuring methods. In different ways they open opportunities to promote evidence based policy making.

We would like to acknowledge the collaborative efforts of the Associate Editors to this special issue: Jonathan Rosenhead, John Mingers, Fran Ackermann, Keith Hipel, Dorien DeTombe, Cláudio Thomaz Bornstein, René Victor Valqui Vidal, Per Sigurd Agrell, Paulo de Martino Jannuzzi, Roberto dos Santos Bartholo, Luiz Fernando Loureiro Legey, Alberto Gabbay Canen, Maria Stella de Castro Lobo and Denis Bouyssou. We are especially grateful to the editor-in-chief Horacio Hideki Yanasse for his supportive and encouraging attitude along the entire editorial job.

Guest Editor: Marcos Pereira Estellita Lins, Production Engineering Program, Federal University of Rio de Janeiro. 